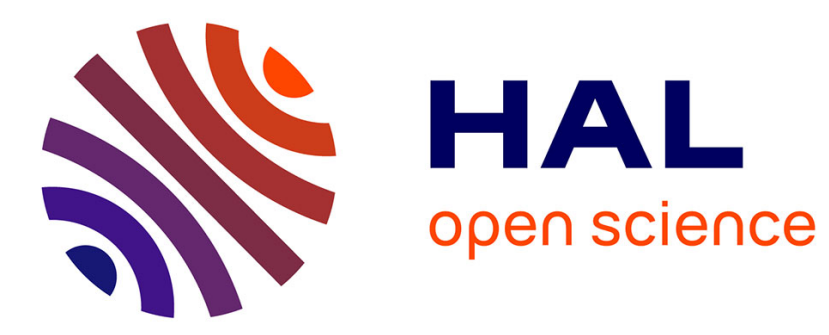

\title{
Formal Information Model for Representing Production Resources
}

\author{
Niko Siltala, Eeva Järvenpää, Minna Lanz
}

\section{To cite this version:}

Niko Siltala, Eeva Järvenpää, Minna Lanz. Formal Information Model for Representing Production Resources. IFIP International Conference on Advances in Production Management Systems (APMS), Sep 2016, Iguassu Falls, Brazil. pp.53-60, 10.1007/978-3-319-51133-7_7 . hal-01615699

\section{HAL Id: hal-01615699 \\ https://hal.inria.fr/hal-01615699}

Submitted on 12 Oct 2017

HAL is a multi-disciplinary open access archive for the deposit and dissemination of scientific research documents, whether they are published or not. The documents may come from teaching and research institutions in France or abroad, or from public or private research centers.
L'archive ouverte pluridisciplinaire HAL, est destinée au dépôt et à la diffusion de documents scientifiques de niveau recherche, publiés ou non, émanant des établissements d'enseignement et de recherche français ou étrangers, des laboratoires publics ou privés. 


\title{
Formal Information Model for Representing Production Resources
}

\author{
Niko Siltala, Eeva Järvenpää, and Minna Lanz \\ Tampere University of Technology, Department of Mechanical Engineering and \\ Industrial Systems, Tampere, Finland \\ [niko.siltala, eeva.jarvenpaa, minna.lanz]@tut.fi
}

\begin{abstract}
This paper introduces a concept and associated descriptions to formally describe physical production resources for modular and reconfigurable production systems. These descriptions are source of formal information for (automatic) production system design and (re-)configuration. They can be further utilized during the system deployment and execution. The proposed concept and the underlying formal resource description model is composed of three different description levels, namely Abstract Resource Description (ARD), Resource Description (RD) and Resource Instance Description (RID), each having different scope and objectives. This paper discusses in details the content and differences between these description levels.
\end{abstract}

Keywords: production resource model, production resource description, production system design, Reconfigurable Manufacturing System, RMS, XML

\section{Introduction}

The requirements on production systems are continuously being shifted towards higher flexibility and adaptability. Increasing volatility in the economies, shortening innovation and product life cycles, and ever increasing number of variants, call for production systems, which comply with these changing demands. There is a need for rapidly responding production systems that can timely adjust to the required changes in processing functions and production capacity. System reconfiguration is required on three levels: physical, logical and parametric [1] System reconfiguration is the enabler for re-use and sustainability of production resources.

Despite the high efforts towards reconfigurable production systems and standardization activities focusing on unification of mechanical as well as communication and control interfaces, reconfiguration of assembly systems is still rare in real factories. The usual business today, when the product model changes, is to scrap the existing resources and build a new assembly system from a scratch. This is due to high engineering, integration, and programming efforts and skills needed to re-configure the existing system, as well as uncertainties related to the needed effort. One of the main reasons for infeasibility of reconfiguration is 
the lack of sufficient and accurate information about the production resources, and their capabilities associated to the current system, its life cycle, and usage history [2]. In addition to Hardware (HW) and Software (SW) interfaces, efficient methodologies, tools and information models are needed to support planners and engineers in the reconfiguration process, and also to allow logical and parametric reconfiguration to take place autonomously while the system is running.

The European Commission funded project ReCaM [3], started in November 2015 , aims to find solutions for the above mentioned issues. It targets to develop a set of integrated tools for rapid and autonomous reconfiguration of production systems, integrated with the existing production planning and scheduling tools - MES. The ReCaM approach is based on intelligent plug-and-produce capable self-describing Mechatronic Objects (MOs), which are able to auto-program and self-adjust to the required task. The formal resource descriptions are proposed to capture comprehensively and thoroughly the characteristics of a production devices, providing a foundation for rapid creation of new system configurations.

The objective of this paper is to open the insights of the resource description model and its formal resource descriptions. The paper is organized as follows: Section two discusses other existing resource description models. Third section represents the proposed three level resource description model. Finally, section four discusses the future developments and concludes the paper.

\section{Existing Production Resource Descriptions}

There exist some device related descriptions, however having a slightly different focus. Electronic Device Description Language (EDDL) [4-6] and its continuation Field Device Integration (FDI) $[7,8]$ are used to describe process automation components, having focus on lower level elementary components. The various fieldbus device descriptions for e.g. Prodibus/-Net, DeviceNet and EtherCAT, all fall into same category, having focus even lower level components and configurations for control systems.

Contrary, AutomationML [9-11] has focus in system integration and representation. The basic assumption of the AutomationML is that the templates of modules are injected to the system description, but they are always customised and modified extensively for the project's purposes. This means that the modules are not stable and reusable entities, but are engineered or customised over and over again. Furthermore, the concept requires that the internal design and implementation of a module is revealed extensively and completely for the system design. Nevertheless, the generality of the approach brings the expression power for this concept. It is more probable that the same concept can cover all unexpected situations arriving in realistic production system design landscape. In context of AutomationML, there exist some publications representing aspects of re-usable components like [12] with mechatronic units and [13,14] with SmartComponents. These both get close to objectives of the work reported in this paper, having the main difference on harmonisation of capabilities, not having abstraction layer present and encapsulation of resource's Intellectual 


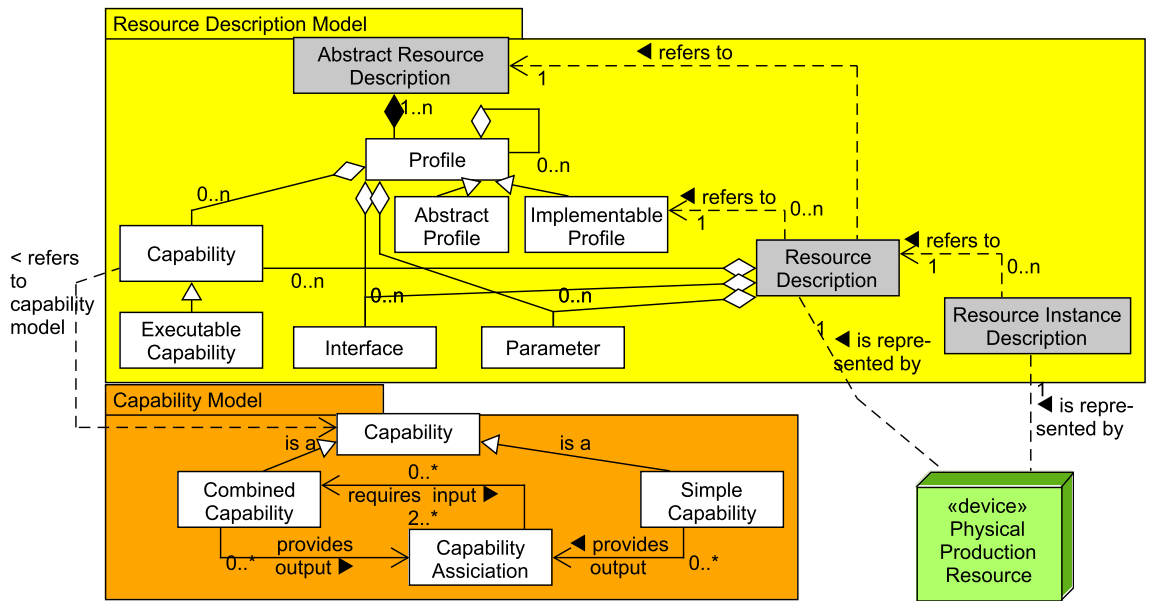

Fig. 1. Main parts of the Resource Description Model and connection to Capability model. A concrete production resource (device) is represented at right bottom.

Properties and implementation. This paper is refining and extending the earlier work reported in [15-17] and summarizes the core models from [18].

\section{Proposed Production Resource Description Model}

Resource description concept is a comprehensive XML-based digital representation of a technical entity. It integrates together information of a production resource related to functional, geometrical, mechanical, communication and control aspects. It allows giving a description of resources' functionality including capabilities; interfaces to other resources; parameters related to business, environment and technical characteristics; and life cycle related information. Resource description concept is a roof term and encapsulates detailed parts of descriptions, namely Abstract Resource Description (ARD), Resource Description (RD) and Resource Instance Description (RID), and their interrelations. Figure 1 describes the relation between the three main parts of the model (Resource Description Model part). In addition, figure shows the link to the Capability Model. The Capability Model, its application, and how it is combined with Resource Description Model are discussed more in details in [19]. Figure 3 provides more detailed view of different resource descriptions and their content.

\subsection{Abstract Resource Description}

Abstract Resource Description (ARD) is an abstraction and a reference model for production resources. It forms an abstract digital specification and generalisation for a collection of similar kind of production resources (e.g. grippers, feeders, 
glue dispensers, welding devices, etc.). In other words, ARD is a generalisation, which can be specialised as a physical production resource. The objective of $\mathrm{ARD}$ is to enable compatibility, interchangeability and connectivity between different production resources, through harmonisation and grouping of interfaces specifications across different types of resources. For example, the task of ARD is to ensure that a robot can be connected to a base, and that a gripper can be connected to a robot, and interchanged with another gripper. The purpose of $\mathrm{ARD}$ is to provide harmonisation over RDs and its content is controlled by a user group(s) or standardisation bodies.

ARD cannot be directly instantiated as a physical resource, but it is composed of one or more Profiles (See Figure 1), which are reflecting production resources. Profile is an integral and inseparable part of ARD and cannot exist alone outside of ARD. Profile defines a reusable construction block of definitions, a structure which is used to specify the detailed section of an ARD. It includes information related to interfaces, capabilities, properties and other features that are composing the generalisation for a set of production resources. This way the information is defined only in one place, which then can be referenced and re-used in other parts of the descriptions. This improves the quality and consistency of the descriptions, as e.g. typing or mishaps related errors can be reduced, consistency of information can be increased, and the maintenance of descriptions is facilitated.

Two kinds of Profiles exist - abstract and implementable ones, but only the latter can be instantiated as RD and its physical implementation. A Profile can be built from $N$ other Profiles with concepts of inheritance or referencing. Figure 2 provides an example of inheritance hierarchy in case of Gripper ARD. The content of Profile characterises the module, and can be used for comparison, evaluation, and selection purposes. Included interfaces and capability definitions are the enablers for mating and fitting the devices together, and making sure that the composition of production modules is able to provide requested functionality for the production task. The interfaces include comprehensive information from mechanical and electrical to service and communication. Generally, it captures all information needed for connecting a production module to other ones or to external world. As a rule, Profiles are introducing the existence and purpose of Properties, but not yet fixing the property values. This provides harmonisation and predictability across the RD definitions, which are later setting the values for these properties.

The Gripper ARD and its Force controlled 2-finger gripper Profile are used as a practical example. It provides an illustration of re-use, construction block behaviour (i.e. define only once), and use of the two types of Profiles. Figure 2 illustrates this example and aforementioned characteristics of Profiles. Each rectangle is a Profile entity. The rectangles with white background and italics are abstract Profiles, of which physical module cannot be created of, and ones with grey background are implementable Profiles, from which the production modules and corresponding RD can be created of. The implementable Profile of our interest is at bottom, highlighted with thicker boarder. The main advantage of Profile concept is coming from inheritance. The Profile Force controlled 2- 


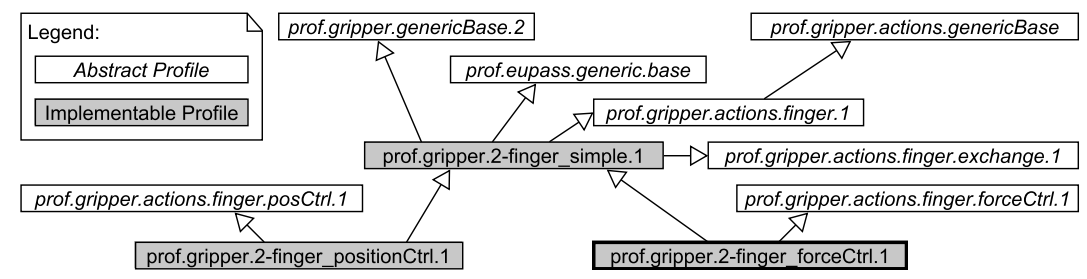

Fig. 2. Example of Profile inheritance

finger gripper could define all its features within a single Profile, but instead it is inheriting features from totally seven other Profiles. Directly it inherits another implementable Profile Simple 2-finger gripper and one abstract Profile prof.gripper.actions.finger.forceCtrl.1. In turn, the Profile Simple 2-finger gripper is inheriting four other abstract Profiles, and so on. The effective advantages are gained when other Profiles are added, like prof.gripper.2-finger_positionCtrl.1.

\subsection{Resource Description}

Resource Description (RD) is a digital representation of a real, physical production resource. It is the main description in this model as it describes the details associated to a specific type of HW resource, used for production as a part of a production system. The description is common to all same kind of resources i.e. resources having the same vendor, model, type and version. It is defined and distributed by the module provider, and it serves both as advertisement and source of detailed information for system integration and resource usage.

$\mathrm{RD}$ represents the catalogue information and a bit more about the resource. First, it contains a reference to the ARD and Profile of which this resource claims to implement. This can be used for validation, interoperability and interchangeability purposes. Secondly, in contrast with ARD, RD provides values for the properties defined in ARD / Profiles. Furthermore, it provides the vendor information; functional description and related parameter values (i.e. relation to Capability Model); physical properties (mass, centre of gravity and energy consumption); technical, business and environmental properties; interface port information including type and gender, spatial location, force and torque limits, and kinematics; CAD models, manuals and documentation; and test and calibration routines. These are illustrated in Figure 3, which provides a detailed view of the three different resource descriptions and their content.

The different RDs are intended to be available on-line, like the ARDs as well. Thus, a web service [20] is made available in order to demonstrate a solution to share and distribute these information, and make searches from it. The web service is discussed in [21]. 


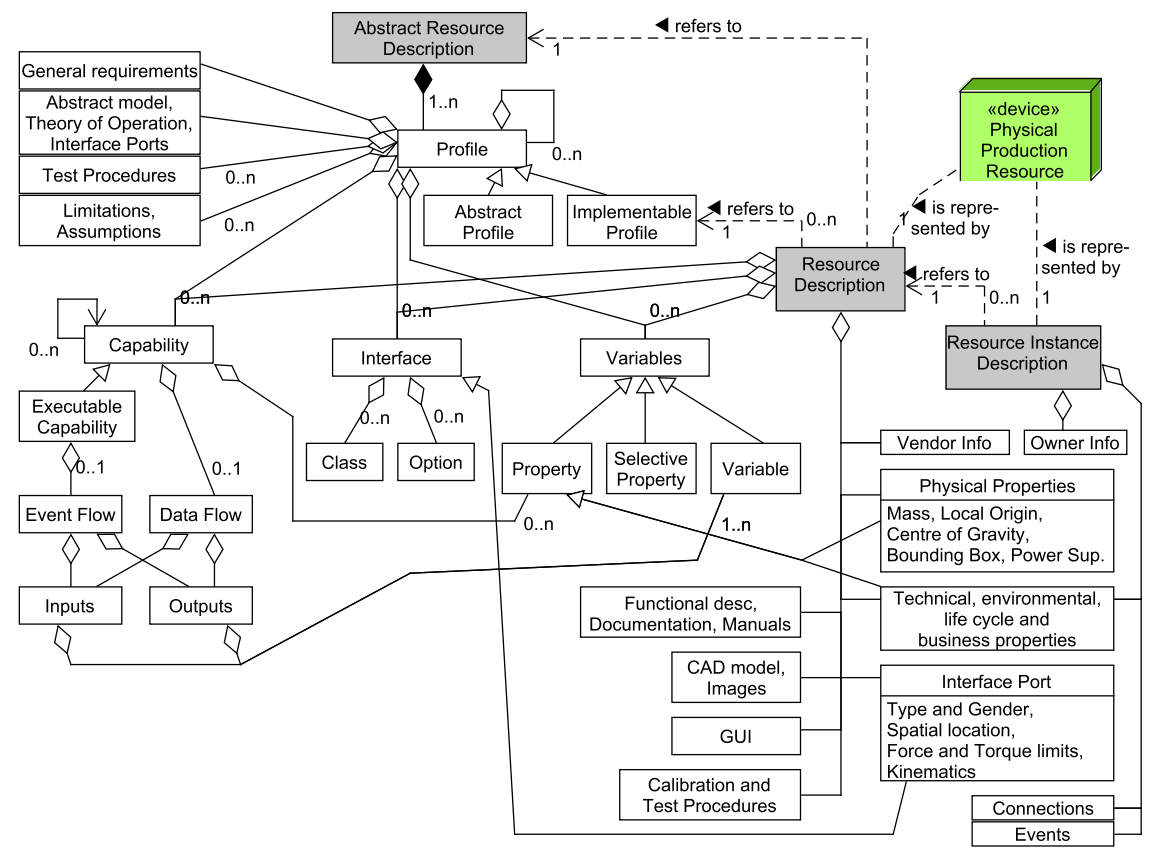

Fig. 3. Resource Description Model

\subsection{Resource Instance Description}

Condition and capabilities of the resources evolve during their individual life cycles and usages. Resource Instance Description (RID) is a digital representation of an individual physical instance of a resource. It carries the resources' current state and historical data events - it is an accumulating information storage. It appends the RD with information that cannot be generalised over all instances of the same resource type, but is specific to one instance only. For instance, if the capability or life cycle parameters (such as Mean Time Between Failure (MTBF) or tolerances) are changed during the resource life cycle, the RID will contain the updated information. The RID should travel all the time with the physical production resource.

\subsection{Example Instances of Resource Description Model}

Table 1 summarises the characteristics of different top level descriptions composing the Resource Description Model, by connecting these with compacted definition and an illustrative example of each description. ARD represents specific technologies such as grippers, axis -systems or feeders, and collect all associated Profiles together. Profile provides generalised specification of specific kind of entities, such as 2-finger grippers. These are the parts composing a single ARD. 
Table 1. Comparison of main entities of Production Resource Model

\begin{tabular}{|c|c|c|}
\hline Entity & Definition & Example \\
\hline$\overline{\mathrm{ARD}}$ & is container for Profiles & Grippers ARD \\
\hline Profile & $\begin{array}{l}\text { is description of abstract module and part of } \\
\text { ARD. }\end{array}$ & Abstraction of a 2-finger gripper \\
\hline $\mathrm{RD}$ & $\begin{array}{l}\text { is digital representation of physical module. } \\
\text { Derived from one Profile }\end{array}$ & $\begin{array}{l}\text { 2-finger gripper from vendor } \mathrm{V}_{A} \\
\mathrm{w} / \text { type: } \mathrm{T} 1\end{array}$ \\
\hline RID & $\begin{array}{l}\text { is digital representation of a specific module } \\
\text { instance. }\end{array}$ & $\begin{array}{l}\text { Type: T1 gripper w/ serial: } \\
\text { SN123 }\end{array}$ \\
\hline
\end{tabular}

$\mathrm{RD}$ turns focus to the module providers (vendor $\mathrm{V}_{A}$ ). They provide a detailed description of their module (third row in Table 1). This description respects the definitions made in aforementioned Profile and ARD. Finally, when vendor $\mathrm{V}_{A}$ produces physical entity of such gripper of type T1, they assign a serial number to this piece of HW and create a RID for it (fourth row in Table 1).

\section{Discussion and Conclusions}

This paper presented an overview of a three level model to describe production resources. This model and descriptions can be utilised in various phases of Reconfigurable Manufacturing System (RMS) production system design, reconfiguration and commissioning. How to apply these models is discussed in [18, Ch.7].

In the due course of the ReCaM project, there are already identified improvements and new requirements related to the production resource descriptions. These are: a) further development, synchronisation, and tighter integration with the Capability model; b) detailed definition of Executable Capability concept, i.e. interfaces for the controls and SW services; c) improvements on mechanical interface description in relation to interfaces connecting the resource to the produced items; d) including generalised Graphical User Interface (GUI) definitions into descriptions, from which resource specific GUIs can be generated automatically; and finally, e) editors for creating and modifying the resource description files. These improvements are in progress. There is interest to research if this model, especially RD, could be translated to format of AutomationML. The presented models and proposed improvements will be validated during the ReCaM project.

\section{Acknowledgement}

This research has received funding from the European Union's Horizon 2020 research and innovation programme under grant agreement no 680759 [3].

\section{References}

1. ElMaraghy, H.A.: Flexible and reconfigurable manufacturing systems paradigms. Int. J. Flex. Manuf. Syst. 17, 261-276 (2005). 
2. Järvenpää, E.: Capability-based Adaptation of Production Systems in a Changing Environment. PhD dissertation. Tampere University of Technology. 190 p. (2012)

3. ReCaM - Rapid Reconfiguration of Flexible Production Systems through Capabilitybased Adaptation, Auto-configuration, and Integrated tools for Production Planning project, EU Horizon 2020, GA No 680759, http://www.recam-project.eu.

4. Merget, O.: The electronic Integration of Field Devices. IEE Comput. Control Eng. $22-23(2003)$.

5. IEC: IEC 61804-3:2006. Function blocks (FB) for process control - Part 3: Electronic Device Description Language (EDDL). (2006).

6. FDI Cooperation: Electronic Device Description Language, http://www.eddl.org/.

7. Neumann, P., Simon, R., Diedrich, C., Riedl, M.: Field device integration. In: 8th International Conference on Emerging Technologies and Factory Automation (ETFA 2001). Proceedings (Cat. No.01TH8597). pp. 63 - 68. IEEE (2001).

8. FDI Cooperation, http://www.fdi-cooperation.com.

9. AutomationML / IEC: IEC 62714-1:2014 Engineering data exchange format for use in industrial automation systems engineering - Automation markup language - Part 1: Architecture and general requirements. (2014).

10. Automation ML, http://www.automationml.org/.

11. Lüder, A., Hundt, L., Keibel, A.: Description of manufacturing processes using AutomationML. In: 2010 IEEE 15th Conference on Emerging Technologies \& Factory Automation (ETFA 2010). 8 p. (2010).

12. Lüder, A., Foehr, L.H.M., Wagner, T., Zaddach, J.-J., Holm, T.: Manufacturing system engineering with mechatronical units. In: IEEE 15th Conference on Emerging Technologies \& Factory Automation (ETFA 2010). 8 p. Bilbao, Spain (2010).

13. Bartelt, M., Schyja, A., Kuhlenkötter, B.: More than a Mockup - SmartComponents: reusable fully functional virtual components from scratch. Prod. Eng. 8, pp. 727-735 (2014).

14. Schyja, A., Bartelt, M., Kuhlenkötter, B.: From Conception Phase up to Virtual Verification Using AutomationML. Procedia CIRP. 23, pp. 171-177 (2014).

15. Siltala, N., Hofmann, A.F., Tuokko, R., Bretthauer, G.: Emplacement and Blue Print-An approach to handle and describe modules for Evolvable Assembly Systems. In: Hideki, H. (ed.) Proc. of 9th International Symposium on Robot Control (SYROCO’09). pp. 86-91. IFAC, Gifu, Japan (2009).

16. Siltala, N., Tuokko, R.: Emplacement and Blue Print - Electronic Module Description Supporting Evolvable Assembly Systems Design, Deployment and Execution. In: Huang, G.Q., Mak, K.L., and Maropoulos, P.G. (eds.) Proc. of 6th CIRP-sponsored International Conference on Digital Enterprise Technology (DET). pp. 773-788. Springer-Verlag Berlin Heidelberg 2010, Hong Kong (2009).

17. Siltala, N., Tuokko, R.: Use of electronic module descriptions for modular and Reconfigurable Assembly Systems. In: 2009 IEEE International Symposium on Assembly and Manufacturing. pp. 214-219. Suwon, Korea (2009).

18. Siltala, N.: Formal Digital Description of Production Equipment Modules for Supporting System Design and Deployment. PhD dissertation. Tampere University of Technology. 211 p. (2016)

19. Järvenpää, E., Siltala, N., Lanz, M.: Formal Resource and Capability Descriptions Supporting Rapid Reconfiguration of Assembly Systems. In: 2016 IEEE International Symposium on Assembly and Manufacturing. Texas, USA (to be published).

20. Emplacement Web Service, http://resourcedescription.tut.fi/EmplacementWS/.

21. Siltala, N., Tuokko, R.: A web tool supporting the management and use of electronic module descriptions for Evolvable Production Systems. In: 2010 IEEE International Symposium on Industrial Electronics. pp. 2641-2646. Bari, Italy, (2010). 